\title{
On Lipschitz continuity with respect to the Poincaré metric of linear contractions between Möbius gyrovector spaces
}

\section{Keiichi Watanabe ${ }^{1 *}$ (D)}

\section{"Correspondence:}

wtnbk@math.sc.niigata-u.ac.jp 'Department of Mathematics, Faculty of Science, Niigata University, Niigata City, 950-2181, Japan

\begin{abstract}
For every linear operator between inner product spaces whose operator norm is less than or equal to one, we show that the restriction to the Möbius gyrovector space is Lipschitz continuous with respect to the Poincaré metric. Moreover, the Lipschitz constant is precisely the operator norm.
\end{abstract}

MSC: Primary 47A30; secondary 20N05; 46C05; 46T99; 51M10; 83A05

Keywords: Linear operator; Contraction; Lipschitz continuous; Gyrovector space

\section{Introduction}

The notion of Lipschitz continuity of mappings between two metric spaces is well known and significant in all fields of mathematics, particularly in geometry and analysis. It is one of the most fundamental facts in functional analysis that an arbitrary bounded linear operator $T$ between normed spaces is Lipschitz continuous. Thus, for all vectors $u, v$ in the normed space on which $T$ is defined, the following norm inequality holds:

$$
\|T u-T v\| \leq\|T\|\|u-v\| .
$$

Moreover, the Lipschitz constant is precisely the operator norm of $T$. That is, the identity

$$
\sup _{u \neq v} \frac{\|T u-T v\|}{\|u-v\|}=\|T\|
$$

holds.

Let $\mathbb{D}=\{z \in \mathbb{C} ;|z|<1\}$ be the open unit disk of the complex number field $\mathbb{C}$. The Möbius addition on $\mathbb{D}$ is defined by the equation

$$
a \oplus b=\frac{a+b}{1+\bar{a} b}
$$

for any $a, b \in \mathbb{D}$, which appears in various branches of mathematics. The Poincaré disk $(\mathbb{D}, \oplus)$ is one of the earliest examples of gyrogroups. The theory of gyrogroups and gy-

c) The Author(s) 2021. This article is licensed under a Creative Commons Attribution 4.0 International License, which permits use, sharing, adaptation, distribution and reproduction in any medium or format, as long as you give appropriate credit to the original author(s) and the source, provide a link to the Creative Commons licence, and indicate if changes were made. The images or other third party material in this article are included in the article's Creative Commons licence, unless indicated otherwise in a credit line to the material. If material is not included in the article's Creative Commons licence and your intended use is not permitted by statutory regulation or exceeds the permitted use, you will need to obtain permission directly from the copyright holder. To view a copy of this licence, visit http://creativecommons.org/licenses/by/4.0/ 
rovector spaces was initiated in connection with Einstein's special theory of relativity and intensively studied by A.A. Ungar. Especially, in [8-10], Ungar established the concept of real inner product gyrovector spaces, including Möbius gyrovector spaces. Let us briefly recall the definition of the Möbius gyrovector spaces. No knowledge of general theory of gyrogroups or gyrovector spaces is required to read this paper except for some basic facts on Möbius addition, Möbius scalar multiplication, and (gyro) distance function; however, it is fundamental for our motivation and background. In addition, notations from gyrovector space theory simplify expressions of our formulae remarkably. For elementary facts on functional analysis and CBS type inequalities, one can refer to [2, 7]. Let $\mathcal{H}$ be a complex inner product space with a positive definite inner product $\left\langle\cdot, \cdot \cdot\right.$, and let $\mathcal{H}_{s}$ be the open $s$-ball of $\mathcal{H}$,

$$
\mathcal{H}_{s}=\{u \in \mathcal{H} ;\|u\|<s\}
$$

for any fixed $s>0$, where $\|u\|=\langle u, u\rangle^{\frac{1}{2}}$. Although definitions and results are often described for real inner product spaces in the literature, they are also valid for complex inner product spaces as far as we are concerned in this paper, with some trivial modifications such as the change from $\langle u, v\rangle$ to $\operatorname{Re}\langle u, v\rangle$.

Definition 1.1 ([11, Definition 3.40, Definition 6.83]) The Möbius addition $\oplus_{M}$ and the Möbius scalar multiplication $\otimes_{M}$ are given by the equations

$$
\begin{aligned}
& u \oplus_{\mathrm{M}} v=\frac{\left(1+\frac{2}{s^{2}} \operatorname{Re}\langle u, v\rangle+\frac{1}{s^{2}}\|v\|^{2}\right) u+\left(1-\frac{1}{s^{2}}\|u\|^{2}\right) v}{1+\frac{2}{s^{2}} \operatorname{Re}\langle u, v\rangle+\frac{1}{s^{4}}\|u\|^{2}\|v\|^{2}}, \\
& r \otimes_{\mathrm{M}} u=s \tanh \left(r \tanh ^{-1} \frac{\|u\|}{s}\right) \frac{u}{\|u\|} \quad(\text { if } u \neq 0), \quad r \otimes_{\mathrm{M}} 0=0
\end{aligned}
$$

for any $u, v \in \mathcal{H}_{s}$ and $r \in \mathbb{R}$. The addition $\oplus_{\mathrm{M}}$ and the scalar multiplication $\otimes_{\mathrm{M}}$ for real numbers are defined by the equations

$$
a \oplus_{\mathrm{M}} b=\frac{a+b}{1+\frac{1}{s^{2}} a b}, \quad r \otimes_{\mathrm{M}} a=s \tanh \left(r \tanh ^{-1} \frac{a}{s}\right)
$$

for any $a, b \in(-s, s)$ and $r \in \mathbb{R}$.

We simply denote $\oplus_{\mathrm{M}}, \otimes_{\mathrm{M}}$ by $\oplus_{s}, \otimes_{s}$, respectively. The ball $\mathcal{H}_{s}$ expands to the whole space $\mathcal{H}$ as the parameter $s \rightarrow \infty$, and each result in linear functional analysis can be recaptured from the counterpart in gyrolinear analysis.

Theorem 1.2 (cf. [11, after Remark 3.41], [10, p. 1054]) The Möbius addition (resp. Möbius scalar multiplication) reduces to the ordinary vector addition (resp. scalar multiplication) as $s \rightarrow \infty$, that is,

$$
\begin{aligned}
& u \oplus_{s} v \rightarrow u+v \quad(s \rightarrow \infty), \\
& r \otimes_{s} u \rightarrow r u \quad(s \rightarrow \infty)
\end{aligned}
$$

for any $u, v \in \mathcal{H}$ and $r \in \mathbb{R}$. 
Definition 1.3 ([11, (2.1), (6.286), (6.293)]) The inverse element of $u$ with respect to $\oplus_{s}$ obviously coincides with $-u$. We use the notation

$$
u \ominus_{s} v=u \oplus_{s}(-v)
$$

as in vector spaces. Moreover, the Möbius gyrodistance function $d$ and Poincaré distance function (or Möbius metric) $h$ are defined by the equations

$$
\begin{aligned}
& d(u, v)=\left\|v \ominus_{s} u\right\|, \\
& h(u, v)=\tanh ^{-1} \frac{d(u, v)}{s} .
\end{aligned}
$$

Theorem 1.4 (cf. [11, (6.294)]. See also [4, 19]) The Poincaré distance function $h$ satisfies the triangle inequality, so that $\left(\mathcal{H}_{s}, h\right)$ is a metric space. In addition, if $\mathcal{H}$ is a Hilbert space, then $\left(\mathcal{H}_{s}, h\right)$ is complete as a metric space.

In recent years, various notions in the Möbius gyrovector spaces have been established as counterparts to those in Hilbert spaces, such as orthogonal gyrodecomposition with respect to closed gyrovector subspaces, orthogonal gyroexpansion with respect to orthogonal bases, Cauchy-Schwarz type inequalities, and continuous quasi gyrolinear functionals (cf. [1, 12-18]). The Cauchy-Bunyakovsky-Schwarz (CBS in the sequel) inequality is one of the most fundamental inequalities in mathematics. Recently, a CBS type inequality related to the Möbius operations was obtained.

Theorem 1.5 (cf. [16, Theorem 3.6, Theorem 3.7], [17, Theorem 15]) For any $u, v \in \mathcal{H}$, $s>\max \{\|u\|,\|v\|\}$ and $w \in \mathcal{H}$ with $\|w\| \leq 1$, the following inequality holds:

$$
h(\langle u, w\rangle,\langle v, w\rangle) \leq\|w\| h(u, v)
$$

or

$$
\left|\langle u, w\rangle \ominus_{s}\langle v, w\rangle\right| \leq\|w\| \otimes_{s}\left\|u \ominus_{s} v\right\|
$$

Moreover, for any $s>0$ and $w \in \mathcal{H}$ with $\|w\| \leq 1$, the following identity holds:

$$
\sup _{\|u\|,\|v\|<s, u \neq v} \frac{h(\langle u, w\rangle,\langle v, w\rangle)}{h(u, v)}=\|w\| .
$$

This result implies that every linear functional $u \mapsto\langle u, w\rangle$ with $\|w\| \leq 1$ is Lipschitz continuous on the Möbius gyrovector space with respect to the Poincaré metric, and that the Lipschitz constant is precisely $\|w\|$. It is so desirable to extend this result of linear functionals to linear operators with norm less than or equal to one. Let $\mathcal{H}, \mathcal{K}$ be inner product spaces, let $s, s^{\prime}>0$, and let $T$ be a bounded linear operator from $\mathcal{H}$ into $\mathcal{K}$. Suppose that $\|T\| \leq \frac{s^{\prime}}{s}$. Then, it is obvious that the restriction of $T$ maps $\mathcal{H}_{s}$ into $\mathcal{K}_{s^{\prime}}$, and one can expect that they form one of the most fundamental classes of mappings between the Möbius gyrovector spaces. Although the restriction of bounded linear operators does not preserve the Möbius addition and the Möbius scalar multiplication in general, they can be 
considered as the most natural counterpart to bounded linear operators between Hilbert spaces. Maps that preserve gyro addition on gyrovector spaces are known to be special in a sense (cf. [5, Theorem 1], [3, Theorem 6], [17, Theorem 11]). Assume $s=s^{\prime}$ for simplicity. In this article, for every linear operator between inner product spaces whose operator norm is less than or equal to one, we show that the restriction to the Möbius gyrovector space is Lipschitz continuous with respect to the Poincaré metric. Moreover, the Lipschitz constant is precisely the operator norm. In addition, the classical formula (1.1) can be recaptured from our novel result by letting $s \rightarrow \infty$.

The main result in this article is Theorem 3.3, which is a satisfactory extension of [16, Theorem 3.6]. Also the core of Theorem 3.3 is Theorem 3.2, which is an extension of [15, Theorem 5] to an operator version. Although the proofs have similarity to the corresponding one in [15] or [16], we present them for the sake of completeness and reader's convenience. The technical ingredient in this paper is the use of an inequality derived from the classical CBS inequality, and the rest of the proofs are just refinement and conversion to the operator version of those in [15] and [16].

\section{Preliminaries}

In this section, we collect some necessary results. The following lemma is an easy consequence of the definition. One can refer to [12, Proposition 2.3], [13, Lemma 12, Lemma 14(i)].

Lemma 2.1 Let $s>0$. The following formulae hold:

(i) $\left\|u \oplus_{s} v\right\|^{2}=\frac{\|u\|^{2}+2 \operatorname{Re}\langle u, v\rangle+\|v\|^{2}}{1+\frac{2}{s^{2}} \operatorname{Re}\langle u, v\rangle+\frac{1}{s^{4}}\|u\|^{2}\|v\|^{2}}$

(ii) $\frac{u}{s} \oplus_{1} \frac{v}{s}=\frac{u \oplus_{s} v}{s}$

(iii) $r \otimes_{1} \frac{u}{s}=\frac{r \otimes_{s} u}{s}$

for any $u, v \in \mathcal{H}_{s}$ and $r \in \mathbb{R}$.

Note that the Möbius operations generally are not commutative, associative, or distributive. Furthermore, the ordinary scalar multiplication does not distribute the Möbius addition. However, the restricted Möbius operations to the interval $(-s, s)$ together with the ordinary addition and multiplication have a familiar nature.

Lemma 2.2 The following identities hold:

$$
\begin{aligned}
& a \oplus_{s} b=b \oplus_{s} a, \\
& a \oplus_{s}\left(b \oplus_{s} c\right)=\left(a \oplus_{s} b\right) \oplus_{s} c, \\
& 0 \oplus_{s} a=a \oplus_{s} 0=a, \quad(-a) \oplus_{s} a=a \oplus_{s}(-a)=0, \\
& 1 \otimes_{s} a=a, \\
& \left(r_{1} r_{2}\right) \otimes_{s} a=r_{1} \otimes_{s}\left(r_{2} \otimes_{s} a\right), \\
& \left(r_{1}+r_{2}\right) \otimes_{s} a=r_{1} \otimes_{s} a \oplus_{s} r_{2} \otimes_{s} a, \\
& r \otimes_{s}\left(a \oplus_{s} b\right)=r \otimes_{s} a \oplus_{s} r \otimes_{s} b
\end{aligned}
$$

for any $a, b, c \in(-s, s), r_{1}, r_{2}, r \in \mathbb{R}$. 
In addition, we need the following lemma.

Lemma 2.3 ([16, Lemma 1.5]) If $-s<b_{j} \leq a_{j}<s(j=1,2)$, then

$$
b_{1} \oplus_{s} b_{2} \leq a_{1} \oplus_{s} a_{2} .
$$

The equality holds if and only if $a_{j}=b_{j}(j=1,2)$.

The following theorem is crucial when we extend a particular case of the main theorem to the full strength with the Möbius scalar multiplication. The proof is essentially already given in [16, Theorem 3.1].

Theorem 2.4 Let $a, b, p$, and $q$ be real numbers with $0 \leq a, b, p, q \leq 1$. If t is a real number with $|t| \leq 1$, then the following inequality holds:

$$
\begin{aligned}
(p+q) & \sqrt{1-\frac{(p+q)^{2}}{2} a b t+\frac{(p+q)^{4}}{16} a^{2} b^{2}} \\
& \cdot\left\{\sqrt{1-2 p^{2} a b t+p^{4} a^{2} b^{2}} \sqrt{1-2 q^{2} a b t+q^{4} a^{2} b^{2}}+p q\left(a^{2}-2 a b t+b^{2}\right)\right\} \\
\leq & \left\{p \sqrt{1-2 q^{2} a b t+q^{4} a^{2} b^{2}}+q \sqrt{1-2 p^{2} a b t+p^{4} a^{2} b^{2}}\right\} \\
& \cdot\left\{1-\frac{(p+q)^{2}}{2} a b t+\frac{(p+q)^{4}}{16} a^{2} b^{2}+\frac{(p+q)^{2}}{4}\left(a^{2}-2 a b t+b^{2}\right)\right\} .
\end{aligned}
$$

The equality holds if and only if one of the following conditions is satisfied:

(i) $p=q$

(ii) $a=b=0$.

Proof Put $\alpha=a, \theta=\arccos t, \beta=b e^{i \theta}$ and apply [16, Theorem 3.1].

\section{Lipschitz continuity of linear contractions}

Let $\mathcal{H}, \mathcal{K}$ be complex inner product spaces, let $u, v$ be elements in $\mathcal{H}$, and let $T$ be a bounded linear operator from $\mathcal{H}$ into $\mathcal{K}$, unless otherwise stated.

Lemma 3.1 If $u, v \in \mathcal{H}$ and $\|T\| \leq 1$, then the following inequality holds:

$$
|\langle u, v\rangle-\langle T u, T v\rangle| \leq \sqrt{\left(\|u\|^{2}-\|T u\|^{2}\right)\left(\|v\|^{2}-\|T v\|^{2}\right)} .
$$

Proof Although this assertion is almost trivial, we give a proof for the sake of completeness and readers' convenience. Considering the completion of inner product spaces if necessary, we can use the positive semidefinite square root of $I-T^{*} T$, where $I$ denotes the identity operator on $\mathcal{H}$. By the CBS inequality, we obtain

$$
\begin{aligned}
& |\langle u, v\rangle-\langle T u, T v\rangle| \\
& \quad=\left|\left\langle\left(I-T^{*} T\right) u, v\right\rangle\right|=\left|\left\langle\left(I-T^{*} T\right)^{\frac{1}{2}} u,\left(I-T^{*} T\right)^{\frac{1}{2}} v\right\rangle\right| \\
& \quad \leq\left\|\left(I-T^{*} T\right)^{\frac{1}{2}} u\right\|\left\|\left(I-T^{*} T\right)^{\frac{1}{2}} v\right\|=\sqrt{\left(\|u\|^{2}-\|T u\|^{2}\right)\left(\|v\|^{2}-\|T v\|^{2}\right)} .
\end{aligned}
$$

This completes the proof. 
The following theorem can be considered as a core of the main theorem in the present article, and it is an extension of [15, Theorem 5] to an operator version. It is necessary to consider the polar forms of complex numbers $\langle u, v\rangle$ and $\langle T u, T v\rangle$. We reduce the proof of inequality (3.2) related with contractive linear operators between possibly infinite dimensional inner product spaces to a problem of constrained minimum value of a function of eight real variables $a, b, c, d, \rho, r, t, x$. The tuple $(a, b, c, d, \rho, r, t, x)$ does not move all over the entire a priori rectangular, but is constrained by conditions derived from the norms of $u$ and $v$, the linearity, and contractivity of $T$. We extract constraints (3.3) by the previous lemma.

Theorem 3.2 If $\|u\|,\|v\|<1$ and $\|T\| \leq 1$, then the following inequality holds:

$$
\frac{\|T u\|^{2}-2 \operatorname{Re}\langle T u, T v\rangle+\|T v\|^{2}}{1-2 \operatorname{Re}\langle T u, T v\rangle+\|T u\|^{2}\|T v\|^{2}} \leq \frac{\|u\|^{2}-2 \operatorname{Re}\langle u, v\rangle+\|v\|^{2}}{1-2 \operatorname{Re}\langle u, v\rangle+\|u\|^{2}\|v\|^{2}} .
$$

The equality holds if and only if one of the following conditions is satisfied:

(i) $u=v$

(ii) $\|T u\|=\|u\|$ and $\|T v\|=\|v\|$.

Proof Put

$$
a=\|u\|, \quad b=\|v\|, \quad c=\|T u\|, \quad \text { and } \quad d=\|T v\| .
$$

We can take real numbers $0 \leq \rho, r \leq 1$ and $0 \leq t, x<2 \pi$ such that

$$
\langle u, v\rangle=a b \rho e^{i t} \quad \text { and } \quad\langle T u, T v\rangle=c d r e^{i x} .
$$

Then it follows from (3.1) that

$$
\begin{aligned}
|a b \rho \cos t-c d r \cos x| & \leq\left|a b \rho e^{i t}-c d r e^{i x}\right|=|\langle u, v\rangle-\langle T u, T v\rangle| \\
& \leq \sqrt{\left(\|u\|^{2}-\|T u\|^{2}\right)\left(\|v\|^{2}-\|T v\|^{2}\right)}=\sqrt{\left(a^{2}-c^{2}\right)\left(b^{2}-d^{2}\right)} .
\end{aligned}
$$

Hence, we can obtain

$$
a b \rho \cos t-\sqrt{\left(a^{2}-c^{2}\right)\left(b^{2}-d^{2}\right)} \leq c d r \cos x .
$$

In order to prove inequality (3.2), it is necessary and sufficient to show

$$
\begin{aligned}
& \left(a^{2}-2 a b \rho \cos t+b^{2}\right)\left(1-2 c d r \cos x+c^{2} d^{2}\right) \\
& \quad-\left(1-2 a b \rho \cos t+a^{2} b^{2}\right)\left(c^{2}-2 c d r \cos x+d^{2}\right) \geq 0 .
\end{aligned}
$$

The left-hand side of (3.4) can be calculated as follows:

$$
\begin{aligned}
a^{2}- & 2 a^{2} c d r \cos x+a^{2} c^{2} d^{2} \\
& -2 a b \rho \cos t+4 a b c d \rho r \cos t \cos x-2 a b c^{2} d^{2} \rho \cos t
\end{aligned}
$$




$$
\begin{aligned}
& +b^{2}-2 b^{2} c d r \cos x+b^{2} c^{2} d^{2} \\
& -c^{2}+2 c d r \cos x-d^{2} \\
& +2 a b c^{2} \rho \cos t-4 a b c d \rho r \cos t \cos x+2 a b d^{2} \rho \cos t \\
& -a^{2} b^{2} c^{2}+2 a^{2} b^{2} c d r \cos x-a^{2} b^{2} d^{2} \\
= & a^{2}+a^{2} c^{2} d^{2}+b^{2}+b^{2} c^{2} d^{2}-c^{2}-d^{2}-a^{2} b^{2} c^{2}-a^{2} b^{2} d^{2} \\
& -2 a b \rho \cos t\left(1-c^{2}\right)\left(1-d^{2}\right) \\
& +2 c d r \cos x\left(1-a^{2}\right)\left(1-b^{2}\right) .
\end{aligned}
$$

By using (3.3), we can continue to estimate:

$$
\begin{aligned}
\geq & a^{2}+a^{2} c^{2} d^{2}+b^{2}+b^{2} c^{2} d^{2}-c^{2}-d^{2}-a^{2} b^{2} c^{2}-a^{2} b^{2} d^{2} \\
& -2 a b \rho \cos t\left(1-c^{2}\right)\left(1-d^{2}\right) \\
& +2\left\{a b \rho \cos t-\sqrt{\left(a^{2}-c^{2}\right)\left(b^{2}-d^{2}\right)}\right\}\left(1-a^{2}\right)\left(1-b^{2}\right) \\
= & a^{2}+a^{2} c^{2} d^{2}+b^{2}+b^{2} c^{2} d^{2}-c^{2}-d^{2}-a^{2} b^{2} c^{2}-a^{2} b^{2} d^{2} \\
& -2 a b \rho \cos t\left\{\left(1-c^{2}\right)\left(1-d^{2}\right)-\left(1-a^{2}\right)\left(1-b^{2}\right)\right\} \\
& -2 \sqrt{\left(a^{2}-c^{2}\right)\left(b^{2}-d^{2}\right)\left(1-a^{2}\right)\left(1-b^{2}\right)} \\
\geq & a^{2}+a^{2} c^{2} d^{2}+b^{2}+b^{2} c^{2} d^{2}-c^{2}-d^{2}-a^{2} b^{2} c^{2}-a^{2} b^{2} d^{2} \\
& -2 a b\left\{\left(1-c^{2}\right)\left(1-d^{2}\right)-\left(1-a^{2}\right)\left(1-b^{2}\right)\right\} \\
& -2 \sqrt{\left(a^{2}-c^{2}\right)\left(b^{2}-d^{2}\right)}\left(1-a^{2}\right)\left(1-b^{2}\right) .
\end{aligned}
$$

It follows from arithmetic-geometric mean inequality that

$$
\begin{aligned}
\geq & a^{2}+a^{2} c^{2} d^{2}+b^{2}+b^{2} c^{2} d^{2}-c^{2}-d^{2}-a^{2} b^{2} c^{2}-a^{2} b^{2} d^{2} \\
& -2 a b\left\{\left(1-c^{2}\right)\left(1-d^{2}\right)-\left(1-a^{2}\right)\left(1-b^{2}\right)\right\} \\
& -\left\{\left(a^{2}-c^{2}\right)+\left(b^{2}-d^{2}\right)\right\}\left(1-a^{2}\right)\left(1-b^{2}\right) \\
= & (a-b)^{2}\left\{\left(1-c^{2}\right)\left(1-d^{2}\right)-\left(1-a^{2}\right)\left(1-b^{2}\right)\right\} \\
\geq & 0 .
\end{aligned}
$$

Thus the desired inequality (3.4) is shown, and so inequality (3.2) holds.

It is obvious that condition (i) implies the trivial equality in (3.2). Suppose that condition (ii) is satisfied. If $u=0$, then the equality in (3.2) holds as $\|T v\|=\|v\|$, so we may assume $u \neq 0$. We can obtain

$$
\|u\|^{2}=\|T u\|^{2}=\left\langle T^{*} T u, u\right\rangle \leq\left\|T^{*} T u\right\|\|u\| \leq\|u\|^{2} .
$$

By the equality condition of the CBS inequality, it is easy to see $T^{*} T u=u$. Hence we have

$$
\langle T u, T v\rangle=\left\langle T^{*} T u, v\right\rangle=\langle u, v\rangle .
$$


Condition (ii) and formula (3.5) clearly imply the equality in (3.2). Conversely, suppose that the equality in (3.2) holds. If $u=0$ or $v=0$, then condition (ii) is satisfied. If $u, v \neq 0$, then the proof of inequality (3.4) shows that the following conditions (3.6)-(3.7) hold:

$$
\begin{aligned}
& (\rho \cos t-1)\left\{\left(1-c^{2}\right)\left(1-d^{2}\right)-\left(1-a^{2}\right)\left(1-b^{2}\right)\right\}=0, \\
& a^{2}-c^{2}=b^{2}-d^{2} .
\end{aligned}
$$

By formula (3.6), we have

$$
\left(1-c^{2}\right)\left(1-d^{2}\right)-\left(1-a^{2}\right)\left(1-b^{2}\right)=0 \quad \text { or } \quad \rho \cos t=1 .
$$

If $\left(1-c^{2}\right)\left(1-d^{2}\right)-\left(1-a^{2}\right)\left(1-b^{2}\right)=0$, then $a=c$ and $b=d$, so condition (ii) is satisfied. If $\rho \cos t=1$, then $\langle u, v\rangle=\|u\|\|v\|$, and it follows that $v=\lambda u$ for some positive real number $\lambda$. Therefore, from formula (3.7) we obtain

$$
b^{2}-d^{2}=\|v\|^{2}-\|T v\|^{2}=\lambda^{2}\left(\|u\|^{2}-\|T u\|^{2}\right)=\lambda^{2}\left(a^{2}-c^{2}\right)=\lambda^{2}\left(b^{2}-d^{2}\right) .
$$

The case $b^{2}-d^{2}=0$ leads to condition (ii). The case $b^{2}-d^{2} \neq 0$ yields $\lambda=1$ or condition (i). This completes the proof.

The following theorem can be regarded as the most natural counterpart to the classical norm inequality (1.1) for mappings between Möbius gyrovector spaces obtained by the restriction of contractive linear operators between inner product spaces. Actually, they are Lipschitz continuous with respect to the Poincaré metric. In the rest of the paper, we simply denote $\oplus_{1}, \otimes_{1}$ by $\oplus, \otimes$, respectively.

Theorem 3.3 If s $>\max \{\|u\|,\|v\|\}$ and $\|T\| \leq 1$, then the following inequality holds:

$$
h(T u, T v) \leq\|T\| h(u, v) .
$$

That is,

$$
\left\|T u \ominus_{s} T v\right\| \leq\|T\| \otimes_{s}\left\|u \ominus_{s} v\right\|
$$

or

$$
\sqrt{\frac{\|T u\|^{2}-2 \operatorname{Re}\langle T u, T v\rangle+\|T v\|^{2}}{1-\frac{2}{s^{2}} \operatorname{Re}\langle T u, T v\rangle+\frac{1}{s^{4}}\|T u\|^{2}\|T v\|^{2}}} \leq\|T\| \otimes_{s} \sqrt{\frac{\|u\|^{2}-2 \operatorname{Re}\langle u, v\rangle+\|v\|^{2}}{1-\frac{2}{s^{2}} \operatorname{Re}\langle u, v\rangle+\frac{1}{s^{4}}\|u\|^{2}\|v\|^{2}}} .
$$

The equality holds if and only if one of the following conditions is satisfied:

(i) $u=v$,

(ii) $T=0$,

(iii) $\|T u\|=\|u\|$ and $\|T v\|=\|v\|$.

Proof At first, we show that if $s=1,\|u\|,\|v\|<1$, and $\|T\| \leq 1$, then

$$
\sqrt{\frac{\|T u\|^{2}-2 \operatorname{Re}\langle T u, T v\rangle+\|T v\|^{2}}{1-2 \operatorname{Re}\langle T u, T v\rangle+\|T u\|^{2}\|T v\|^{2}}} \leq\|T\| \otimes \sqrt{\frac{\|u\|^{2}-2 \operatorname{Re}\langle u, v\rangle+\|v\|^{2}}{1-2 \operatorname{Re}\langle u, v\rangle+\|u\|^{2}\|v\|^{2}}} .
$$


The proof uses an argument in [6] that shows the operator monotonicity of the functions $t^{p}$ with $0 \leq p \leq 1$. Let $E$ denote the set of all real numbers $p$ in the interval $[0,1]$ for which if $T: \mathcal{H} \rightarrow \mathcal{K}$ is a bounded linear operator with $\|T\|=p$, then inequality (3.9) holds. Trivially $0 \in E$, and the previous theorem implies that $1 \in E$. It is easy to check that $E$ is a closed subset of $[0,1]$. In order to complete the proof of inequality (3.9), it suffices to show that $E$ is convex. Suppose $p, q \in E$. For an arbitrary bounded linear operator $T: \mathcal{H} \rightarrow \mathcal{K}$ with $\|T\|=\frac{p+q}{2}$, put $T_{1}=\frac{2 p}{p+q} T, T_{2}=\frac{2 q}{p+q} T$. Then $\left\|T_{1}\right\|=p,\left\|T_{2}\right\|=q$, so we have

$$
\begin{aligned}
& \sqrt{\frac{\left\|T_{1} u\right\|^{2}-2 \operatorname{Re}\left\langle T_{1} u, T_{1} v\right\rangle+\left\|T_{1} v\right\|^{2}}{1-2 \operatorname{Re}\left\langle T_{1} u, T_{1} v\right\rangle+\left\|T_{1} u\right\|^{2}\left\|T_{1} v\right\|^{2}}} \leq p \otimes \sqrt{\frac{\|u\|^{2}-2 \operatorname{Re}\langle u, v\rangle+\|v\|^{2}}{1-2 \operatorname{Re}\langle u, v\rangle+\|u\|^{2}\|v\|^{2}}}, \\
& \sqrt{\frac{\left\|T_{2} u\right\|^{2}-2 \operatorname{Re}\left\langle T_{2} u, T_{2} v\right\rangle+\left\|T_{2} v\right\|^{2}}{1-2 \operatorname{Re}\left\langle T_{2} u, T_{2} v\right\rangle+\left\|T_{2} u\right\|^{2}\left\|T_{2} v\right\|^{2}}} \leq q \otimes \sqrt{\frac{\|u\|^{2}-2 \operatorname{Re}\langle u, v\rangle+\|v\|^{2}}{1-2 \operatorname{Re}\langle u, v\rangle+\|u\|^{2}\|v\|^{2}}}
\end{aligned}
$$

We have to show

$$
\sqrt{\frac{\|T u\|^{2}-2 \operatorname{Re}\langle T u, T v\rangle+\|T v\|^{2}}{1-2 \operatorname{Re}\langle T u, T v\rangle+\|T u\|^{2}\|T v\|^{2}}} \leq \frac{p+q}{2} \otimes \sqrt{\frac{\|u\|^{2}-2 \operatorname{Re}\langle u, v\rangle+\|v\|^{2}}{1-2 \operatorname{Re}\langle u, v\rangle+\|u\|^{2}\|v\|^{2}}} .
$$

By Lemma 2.2 and Lemma 2.3, we obtain

$$
\begin{aligned}
& \frac{p+q}{2} \otimes \sqrt{\frac{\|u\|^{2}-2 \operatorname{Re}\langle u, v\rangle+\|v\|^{2}}{1-2 \operatorname{Re}\langle u, v\rangle+\|u\|^{2}\|v\|^{2}}} \\
&= \frac{1}{2} \otimes\left(p \otimes \sqrt{\frac{\|u\|^{2}-2 \operatorname{Re}\langle u, v\rangle+\|v\|^{2}}{1-2 \operatorname{Re}\langle u, v\rangle+\|u\|^{2}\|v\|^{2}}}\right) \\
& \oplus \frac{1}{2} \otimes\left(q \otimes \sqrt{\frac{\|u\|^{2}-2 \operatorname{Re}\langle u, v\rangle+\|v\|^{2}}{1-2 \operatorname{Re}\langle u, v\rangle+\|u\|^{2}\|v\|^{2}}}\right) \\
& \geq \frac{1}{2} \otimes \sqrt{\frac{\left\|T_{1} u\right\|^{2}-2 \operatorname{Re}\left\langle T_{1} u, T_{1} v\right\rangle+\left\|T_{1} v\right\|^{2}}{1-2 \operatorname{Re}\left\langle T_{1} u, T_{1} v\right\rangle+\left\|T_{1} u\right\|^{2}\left\|T_{1} v\right\|^{2}}} \\
& \oplus \frac{1}{2} \otimes \sqrt{\frac{\left\|T_{2} u\right\|^{2}-2 \operatorname{Re}\left\langle T_{2} u, T_{2} v\right\rangle+\left\|T_{2} v\right\|^{2}}{1-2 \operatorname{Re}\left\langle T_{2} u, T_{2} v\right\rangle+\left\|T_{2} u\right\|^{2}\left\|T_{2} v\right\|^{2}}} .
\end{aligned}
$$

Therefore, in order to show (3.10), it is sufficient to prove

$$
\begin{aligned}
2 & \otimes \sqrt{\frac{\|T u\|^{2}-2 \operatorname{Re}\langle T u, T v\rangle+\|T v\|^{2}}{1-2 \operatorname{Re}\langle T u, T v\rangle+\|T u\|^{2}\|T v\|^{2}}} \\
& \leq \sqrt{\frac{\left\|T_{1} u\right\|^{2}-2 \operatorname{Re}\left\langle T_{1} u, T_{1} v\right\rangle+\left\|T_{1} v\right\|^{2}}{1-2 \operatorname{Re}\left\langle T_{1} u, T_{1} v\right\rangle+\left\|T_{1} u\right\|^{2}\left\|T_{1} v\right\|^{2}}} \oplus \sqrt{\frac{\left\|T_{2} u\right\|^{2}-2 \operatorname{Re}\left\langle T_{2} u, T_{2} v\right\rangle+\left\|T_{2} v\right\|^{2}}{1-2 \operatorname{Re}\left\langle T_{2} u, T_{2} v\right\rangle+\left\|T_{2} u\right\|^{2}\left\|T_{2} v\right\|^{2}}} .
\end{aligned}
$$

If $\|T u\|=0$, then inequality (3.11) reduces to the following inequality: 
or

$$
2 \otimes\|T v\| \leq \frac{2 p}{p+q}\|T v\| \oplus \frac{2 q}{p+q}\|T v\|
$$

which is equivalent to

$$
\frac{2\|T v\|}{1+\|T v\|^{2}} \leq \frac{\frac{2 p}{p+q}\|T v\|+\frac{2 q}{p+q}\|T v\|}{1+\frac{2 p}{p+q}\|T \nu\| \cdot \frac{2 q}{p+q}\|T v\|}
$$

The last inequality actually holds by $(p+q)^{2} \geq 4 p q$. So we may assume $\|T u\|,\|T v\| \neq 0$. Put

$$
a=\frac{2}{p+q}\|T u\|, \quad b=\frac{2}{p+q}\|T v\|, \quad t=\frac{4}{(p+q)^{2}} \frac{\operatorname{Re}\langle T u, T v\rangle}{a b} .
$$

Obviously $0<a, b<1$ and $|t| \leq 1$ by the CBS inequality. Furthermore, we have

$$
\begin{array}{llrl}
\|T u\|=\frac{p+q}{2} a, & \|T v\|=\frac{p+q}{2} b, & \operatorname{Re}\langle T u, T v\rangle=\frac{(p+q)^{2}}{4} a b t, \\
\left\|T_{1} u\right\|=p a, & \left\|T_{1} v\right\|=p b, & \operatorname{Re}\left\langle T_{1} u, T_{1} v\right\rangle=p^{2} a b t, \\
\left\|T_{2} u\right\|=q a, & \left\|T_{2} v\right\|=q b, & \operatorname{Re}\left\langle T_{2} u, T_{2} v\right\rangle=q^{2} a b t .
\end{array}
$$

Hence we obtain

$$
\begin{aligned}
2 \otimes \sqrt{\frac{\|T u\|^{2}-2 \operatorname{Re}\langle T u, T v\rangle+\|T v\|^{2}}{1-2 \operatorname{Re}\langle T u, T v\rangle+\|T u\|^{2}\|T v\|^{2}}} & =\frac{2 \sqrt{\frac{\|T u\|^{2}-2 \operatorname{Re}\langle T u, T v\rangle+\|T v\|^{2}}{1-2 \operatorname{Re}\langle T u, T v\rangle+\|T u\|^{2}\|T v\|^{2}}}}{1+\frac{\|T u\|^{2}-2 \operatorname{Re}\langle T u, T v\rangle+\|T v\|^{2}}{1-2 \operatorname{Re}\langle T u, T v\rangle+\|T u\|^{2}\|T v\|^{2}}} \\
& =\frac{(p+q) \sqrt{\frac{a^{2}-2 a b t+b^{2}}{1-\frac{(p+q)^{2}}{2} a b t+\frac{(p+q)^{4}}{16} a^{2} b^{2}}}}{1+\frac{\frac{(p+q)^{2}}{4}\left(a^{2}-2 a b t+b^{2}\right)}{1-\frac{(p+q)^{2}}{2} a b t+\frac{(p+q)^{4}}{16} a^{2} b^{2}}}
\end{aligned}
$$

and

$$
\begin{aligned}
& \sqrt{\frac{\left\|T_{1} u\right\|^{2}-2 \operatorname{Re}\left\langle T_{1} u, T_{1} v\right\rangle+\left\|T_{1} v\right\|^{2}}{1-2 \operatorname{Re}\left\langle T_{1} u, T_{1} v\right\rangle+\left\|T_{1} u\right\|^{2}\left\|T_{1} v\right\|^{2}}} \oplus \sqrt{\frac{\left\|T_{2} u\right\|^{2}-2 \operatorname{Re}\left\langle T_{2} u, T_{2} v\right\rangle+\left\|T_{2} v\right\|^{2}}{1-2 \operatorname{Re}\left\langle T_{2} u, T_{2} v\right\rangle+\left\|T_{2} u\right\|^{2}\left\|T_{2} v\right\|^{2}}} \\
& =\frac{\sqrt{\frac{\left\|T_{1} u\right\|^{2}-2 \operatorname{Re}\left\langle T_{1} u, T_{1} v\right\rangle+\left\|T_{1} v\right\|^{2}}{1-2 \operatorname{Re}\left\langle T_{1} u, T_{1} v\right\rangle+\left\|T_{1} u\right\|^{2}\left\|T_{1} v\right\|^{2}}}+\sqrt{\frac{\left\|T_{2} u\right\|^{2}-2 \operatorname{Re}\left\langle T_{2} u, T_{2} v\right\rangle+\left\|T_{2} v\right\|^{2}}{1-2 \operatorname{Re}\left\langle T_{2} u, T_{2} v\right\rangle+\left\|T_{2} u\right\|^{2}\left\|T_{2} v\right\|^{2}}}}{1+\sqrt{\frac{\left\|T_{1} u\right\|^{2}-2 \operatorname{Re}\left\langle T_{1} u, T_{1} v\right\rangle+\left\|T_{1} v\right\|^{2}}{1-2 \operatorname{Re}\left\langle T_{1} u, T_{1} v\right\rangle+\left\|T_{1} u\right\|^{2}\left\|T_{1} v\right\|^{2}}} \cdot \sqrt{\frac{\left\|T_{2} u\right\|^{2}-2 \operatorname{Re}\left\langle T_{2} u, T_{2} v\right\rangle+\left\|T_{2} v\right\|^{2}}{1-2 \operatorname{Re}\left\langle T_{2} u, T_{2} v\right\rangle+\left\|T_{2} u\right\|^{2}\left\|T_{2} v\right\|^{2}}}} \\
& =\frac{p \sqrt{\frac{a^{2}-2 a b t+b^{2}}{1-2 p^{2} a b t+p^{4} a^{2} b^{2}}}+q \sqrt{\frac{a^{2}-2 a b t+b^{2}}{1-2 q^{2} a b t+q^{4} a^{2} b^{2}}}}{1+p \sqrt{\frac{a^{2}-2 a b t+b^{2}}{1-2 p^{2} a b t+p^{4} a^{2} b^{2}}} \cdot q \sqrt{\frac{a^{2}-2 a b t+b^{2}}{1-2 q^{2} a b t+q^{4} a^{2} b^{2}}}} .
\end{aligned}
$$


Therefore, dividing both sides by $\sqrt{a^{2}-2 a b t+b^{2}}$, our proof of inequality (3.11) is reduced to show that

$$
\begin{aligned}
& \frac{(p+q) \sqrt{1-\frac{(p+q)^{2}}{2} a b t+\frac{(p+q)^{4}}{16} a^{2} b^{2}}}{1-\frac{(p+q)^{2}}{2} a b t+\frac{(p+q)^{4}}{16} a^{2} b^{2}+\frac{(p+q)^{2}}{4}\left(a^{2}-2 a b t+b^{2}\right)} \\
& \leq \frac{p \sqrt{1-2 q^{2} a b t+q^{4} a^{2} b^{2}}+q \sqrt{1-2 p^{2} a b t+p^{4} a^{2} b^{2}}}{\sqrt{1-2 p^{2} a b t+p^{4} a^{2} b^{2}} \sqrt{1-2 q^{2} a b t+q^{4} a^{2} b^{2}}+p q\left(a^{2}-2 a b t+b^{2}\right)},
\end{aligned}
$$

the last inequality actually holds by Theorem 2.4 . Thus the set $E$ is convex, which leads to $E=[0,1]$, so inequality (3.9) holds.

Finally, let $u, v \in \mathcal{H}$ be arbitrary elements, and let $s>\max \{\|u\|,\|v\|\}$. Applying inequality (3.9) to $\frac{u}{s}, \frac{v}{s}$, it is straightforward to deduce inequality (3.8).

For the equality condition, we may assume $s=1$. It is immediate to see that the equality in (3.9) holds provided one of conditions (i), (ii), or (iii) is satisfied.

Conversely, let $\frac{1}{2} \leq p<1,\|u\|,\|v\|<1$, and $\|T\|=p$. Suppose that the equality of (3.9) holds. If we put

$$
p^{\prime}=2 p-1, \quad T_{1}=\frac{2 p^{\prime}}{p^{\prime}+1} T, \quad T_{2}=\frac{2}{p^{\prime}+1} T
$$

then it is obvious that $\left\|T_{1}\right\|=p^{\prime},\left\|T_{2}\right\|=1$. By inequality (3.11), inequality (3.9), and Lemma 2.3, we obtain

$$
\begin{aligned}
& \sqrt{\frac{\|T u\|^{2}-2 \operatorname{Re}\langle T u, T v\rangle+\|T v\|^{2}}{1-2 \operatorname{Re}\langle T u, T v\rangle+\|T u\|^{2}\|T v\|^{2}}} \\
& \leq \frac{1}{2} \otimes \sqrt{\frac{\left\|T_{1} u\right\|^{2}-2 \operatorname{Re}\left\langle T_{1} u, T_{1} v\right\rangle+\left\|T_{1} v\right\|^{2}}{1-2 \operatorname{Re}\left\langle T_{1} u, T_{1} v\right\rangle+\left\|T_{1} u\right\|^{2}\left\|T_{1} v\right\|^{2}}} \\
& \quad \frac{1}{2} \otimes \sqrt{\frac{\left\|T_{2} u\right\|^{2}-2 \operatorname{Re}\left\langle T_{2} u, T_{2} v\right\rangle+\left\|T_{2} v\right\|^{2}}{1-2 \operatorname{Re}\left\langle T_{2} u, T_{2} v\right\rangle+\left\|T_{2} u\right\|^{2}\left\|T_{2} v\right\|^{2}}} \\
& \leq \frac{1}{2} \otimes\left(p^{\prime} \otimes \sqrt{\frac{\|u\|^{2}-2 \operatorname{Re}\langle u, v\rangle+\|v\|^{2}}{1-2 \operatorname{Re}\langle u, v\rangle+\|u\|^{2}\|v\|^{2}}}\right) \\
& \oplus \frac{1}{2} \otimes\left(1 \otimes \sqrt{\frac{\|u\|^{2}-2 \operatorname{Re}\langle u, v\rangle+\|v\|^{2}}{1-2 \operatorname{Re}\langle u, v\rangle+\|u\|^{2}\|v\|^{2}}}\right) \\
& =\frac{p^{\prime}+1}{2} \otimes \sqrt{\frac{\|u\|^{2}-2 \operatorname{Re}\langle u, v\rangle+\|v\|^{2}}{1-2 \operatorname{Re}\langle u, v\rangle+\|u\|^{2}\|v\|^{2}}},
\end{aligned}
$$

where the last formula equals the first one by the assumption. The equality condition of Lemma 2.3 implies

$$
\sqrt{\frac{\left\|T_{2} u\right\|^{2}-2 \operatorname{Re}\left\langle T_{2} u, T_{2} v\right\rangle+\left\|T_{2} v\right\|^{2}}{1-2 \operatorname{Re}\left\langle T_{2} u, T_{2} v\right\rangle+\left\|T_{2} u\right\|^{2}\left\|T_{2} v\right\|^{2}}}=\sqrt{\frac{\|u\|^{2}-2 \operatorname{Re}\langle u, v\rangle+\|v\|^{2}}{1-2 \operatorname{Re}\langle u, v\rangle+\|u\|^{2}\|v\|^{2}}},
$$

which yields one of the following conditions: 
(i) $u=v$,

(ii) $\left\|T_{2} u\right\|=\|u\|$ and $\left\|T_{2} v\right\|=\|v\|$

by the equality condition of Theorem 3.2. We have to consider only the case of condition (ii). The first inequality in the previous series of inequalities also has to be an equality. Namely,

$$
\begin{aligned}
& \sqrt{\frac{\|T u\|^{2}-2 \operatorname{Re}\langle T u, T v\rangle+\|T v\|^{2}}{1-2 \operatorname{Re}\langle T u, T v\rangle+\|T u\|^{2}\|T v\|^{2}}} \\
& =\frac{1}{2} \otimes \sqrt{\frac{\left\|T_{1} u\right\|^{2}-2 \operatorname{Re}\left\langle T_{1} u, T_{1} v\right\rangle+\left\|T_{1} v\right\|^{2}}{1-2 \operatorname{Re}\left\langle T_{1} u, T_{1} v\right\rangle+\left\|T_{1} u\right\|^{2}\left\|T_{1} v\right\|^{2}}} \\
& \oplus \frac{1}{2} \otimes \sqrt{\frac{\left\|T_{2} u\right\|^{2}-2 \operatorname{Re}\left\langle T_{2} u, T_{2} v\right\rangle+\left\|T_{2} v\right\|^{2}}{1-2 \operatorname{Re}\left\langle T_{2} u, T_{2} v\right\rangle+\left\|T_{2} u\right\|^{2}\left\|T_{2} v\right\|^{2}}} .
\end{aligned}
$$

Considering the division by $\sqrt{a^{2}-2 a b t+b^{2}}$ in the proof of (3.11), it yields one of the following conditions:

(i') $p^{\prime}=1$,

(ii') $\|T u\|=\|T v\|=0$,

(iii') $\frac{\operatorname{Re}\langle T u, T v\rangle}{\|T u\|\|T v\|}=1$ and $\|T u\|=\|T v\|$.

However, we have $p^{\prime} \neq 1$, because $p<1$.

Note that, if $\|T u\|=0$, then we have $T_{1} u=T_{2} u=0$ and

$$
\begin{aligned}
\|T v\| & =\frac{1}{2} \otimes\left\|T_{1} v\right\| \oplus \frac{1}{2} \otimes\left\|T_{2} v\right\| \\
& =\frac{1}{2} \otimes \frac{2 p-1}{p}\|T v\| \oplus \frac{1}{2} \otimes \frac{1}{p}\|T v\|,
\end{aligned}
$$

from which we easily obtain $\|T v\|=0$. In case (ii'), obviously $u=v=0$ by $\left\|T_{2} u\right\|=\|u\|$ and $\left\|T_{2} v\right\|=\|v\|$.

In case (iii'), by the equality condition of the CBS inequality, $T v=\lambda T u$ for some positive real number $\lambda$. Hence

$$
\|T u\|=\|T v\|=\lambda\|T u\|
$$

We may assume $\lambda=1$, and it follows that

$$
\langle u, v\rangle=\left\langle T_{2} u, T_{2} v\right\rangle=\frac{1}{p^{2}}\langle T u, T v\rangle=\frac{1}{p^{2}}\|T u\|^{2}=\left\|T_{2} u\right\|\left\|T_{2} v\right\|=\|u\|\|v\| .
$$

Therefore, $v=\mu u$ for some $\mu \geq 0$, and it leads to $u=v$.

Next, suppose $\frac{1}{4} \leq p<\frac{1}{2}$ and that the equality of (3.9) holds for $\|T\|=p$. Put

$$
p^{\prime}=2 p-\frac{1}{2}, \quad T_{1}=\frac{2 p^{\prime}}{p^{\prime}+\frac{1}{2}} T, \quad T_{2}=\frac{2 \cdot \frac{1}{2}}{p^{\prime}+\frac{1}{2}} T .
$$

Then we have $\left\|T_{1}\right\|=p^{\prime},\left\|T_{2}\right\|=\frac{1}{2}$. A similar argument before shows

$$
\sqrt{\frac{\left\|T_{2} u\right\|^{2}-2 \operatorname{Re}\left\langle T_{2} u, T_{2} v\right\rangle+\left\|T_{2} v\right\|^{2}}{1-2 \operatorname{Re}\left\langle T_{2} u, T_{2} v\right\rangle+\left\|T_{2} u\right\|^{2}\left\|T_{2} v\right\|^{2}}}=\frac{1}{2} \otimes \sqrt{\frac{\|u\|^{2}-2 \operatorname{Re}\langle u, v\rangle+\|v\|^{2}}{1-2 \operatorname{Re}\langle u, v\rangle+\|u\|^{2}\|v\|^{2}}},
$$


which yields $u=v$ by the equality condition established just before. This argument can be repeated for half open intervals $\left[1 / 2^{n+1}, 1 / 2^{n}\right)$, and we can conclude that the equality condition holds. This completes the proof.

\section{Remark 3.4}

(i) If $\|T\|=1$, then inequality (3.9) reduces to inequality (3.2).

(ii) The classical norm inequality (1.1) can be recaptured by an argument of elementary calculus and letting $s \rightarrow \infty$ in inequality (3.8).

(iii) Let $w \in \mathcal{H}$ with $\|w\| \leq 1$. If we consider the linear functional defined by

$$
T u=\langle u, w\rangle \quad(u \in \mathcal{H})
$$

then [16, Theorem 3.6] can be regarded as a particular case of Theorem 3.3 in the present paper.

(iv) In equality condition (iii) of Theorem 3.3, it is necessary that $\|T\|=1$ unless $u=v=0$.

The following theorem shows that, for every linear operator between inner product spaces whose operator norm is less than or equal to one, the Lipschitz constant of the restriction to the Möbius gyrovector space is precisely the operator norm if we consider the Poincaré metric.

Theorem 3.5 Let $\mathcal{H}, \mathcal{K}$ be complex inner product spaces, and let $T: \mathcal{H} \rightarrow \mathcal{K}$ be a bounded linear operator with $\|T\| \leq 1$. For any $s>0$, the following identity holds:

$$
\sup _{\|u\|,\|v\|<s, u \neq v} \frac{h(T u, T v)}{h(u, v)}=\|T\| .
$$

Proof It is easy to see that we may assume $s=1$ without loss of generality. Theorem 3.3 implies

$$
\sup _{\|u\|,\|v\|<1, u \neq v} \frac{h(T u, T v)}{h(u, v)} \leq\|T\| .
$$

If we put

$$
K=\sup _{\|u\|,\|v\|<1, u \neq v} \frac{h(T u, T v)}{h(u, v)}
$$

then obviously we have

$$
\sqrt{\frac{\|T u\|^{2}-2 \operatorname{Re}\langle T u, T v\rangle+\|T v\|^{2}}{1-2 \operatorname{Re}\langle T u, T v\rangle+\|T u\|^{2}\|T v\|^{2}}} \leq K \otimes \sqrt{\frac{\|u\|^{2}-2 \operatorname{Re}\langle u, v\rangle+\|v\|^{2}}{1-2 \operatorname{Re}\langle u, v\rangle+\|u\|^{2}\|v\|^{2}}}
$$

for any $u, v \in \mathcal{H}$ with $\|u\|,\|v\|<1$. 
Now, for arbitrary $u, v \in \mathcal{H}$ and $t>\max \{\|u\|,\|v\|\}$, by applying inequality (3.13) to $\frac{u}{t}, \frac{v}{t}$, it is immediate to see

$$
\begin{aligned}
& \sqrt{\frac{\|T u\|^{2}-2 \operatorname{Re}\langle T u, T v\rangle+\|T v\|^{2}}{1-\frac{2}{t^{2}} \operatorname{Re}\langle T u, T v\rangle+\frac{1}{t^{4}}\|T u\|^{2}\|T v\|^{2}}} \\
& \leq t \tanh \left\{K \tanh ^{-1}\left(\frac{1}{t} \sqrt{\frac{\|u\|^{2}-2 \operatorname{Re}\langle u, v\rangle+\|v\|^{2}}{1-\frac{2}{t^{2}} \operatorname{Re}\langle u, v\rangle+\frac{1}{t^{4}}\|u\|^{2}\|v\|^{2}}}\right)\right\} .
\end{aligned}
$$

By letting $t \rightarrow \infty$, we can obtain

$$
\|T u-T v\| \leq K\|u-v\|
$$

for every pair $u, v \in \mathcal{H}$, which implies $\|T\| \leq K$. This completes the proof.

We state the corresponding results in real inner product spaces.

Theorem 3.6 Let $\mathcal{H}, \mathcal{K}$ be real inner product spaces, and let $T: \mathcal{H} \rightarrow \mathcal{K}$ be a bounded real linear operator with $\|T\| \leq 1$. For any $u, v \in \mathcal{H}$ and $s>\max \{\|u\|,\|v\|\}$, the following inequality holds:

$$
h(T u, T v) \leq\|T\| h(u, v) .
$$

That is,

$$
\left\|T u \ominus_{s} T v\right\| \leq\|T\| \otimes_{s}\left\|u \ominus_{s} v\right\|
$$

or

$$
\sqrt{\frac{\|T u\|^{2}-2\langle T u, T v\rangle+\|T v\|^{2}}{1-\frac{2}{s^{2}}\langle T u, T v\rangle+\frac{1}{s^{4}}\|T u\|^{2}\|T v\|^{2}}} \leq\|T\| \otimes_{s} \sqrt{\frac{\|u\|^{2}-2\langle u, v\rangle+\|v\|^{2}}{1-\frac{2}{s^{2}}\langle u, v\rangle+\frac{1}{s^{4}}\|u\|^{2}\|v\|^{2}}} .
$$

The equality holds if and only if one of the following conditions is satisfied:

(i) $u=v$,

(ii) $T=0$,

(iii) $\|T u\|=\|u\|$ and $\|T v\|=\|v\|$.

Theorem 3.7 Let $\mathcal{H}, \mathcal{K}$ be real inner product spaces, and let $T: \mathcal{H} \rightarrow \mathcal{K}$ be a bounded real linear operator with $\|T\| \leq 1$. For any $s>0$, the following identity holds:

$$
\sup _{\|u\|,\|v\|<s, u \neq v} \frac{h(T u, T v)}{h(u, v)}=\|T\| .
$$

\section{Acknowledgements}

This work was also supported by the Research Institute for Mathematical Sciences, a Joint Usage/Research Center located in Kyoto University. It means that the author attended some online conferences by RIMS with no financial support. The author would like to thank the referees and the editor for their valuable comments which improved the original manuscript. 
Funding

This work was partially supported by Grant-in-Aid for Scientific Research(C), JSPS KAKENHI Grant Number JP21 K03288.

Availability of data and materials

Not applicable.

\section{Competing interests}

The author declares that they have no competing interests.

Authors' contributions

The author read and approved the final manuscript.

\section{Publisher's Note}

Springer Nature remains neutral with regard to jurisdictional claims in published maps and institutional affiliations.

Received: 31 May 2021 Accepted: 23 September 2021 Published online: 09 October 2021

\section{References}

1. Abe, T., Watanabe, K.: Finitely generated gyrovector subspaces and orthogonal gyrodecomposition in the Möbius gyrovector space. J. Math. Anal. Appl. 449(1), 77-90 (2017). https://doi.org/10.1016/j.jmaa.2016.11.039

2. Conway, J.B.: A Course in Functional Analysis, 2nd edn. Graduate Texts in Mathematics, vol. 96. Springer, New York (1990)

3. Frenkel, P.E.: On endomorphisms of the Einstein gyrogroup in arbitrary dimension. J. Math. Phys. 57, 032301 (2016)

4. Goebel, K., Reich, S., Uniform Convexity, Hyperbolic Geometry, and Nonexpansive Mappings. Monographs and Textbooks in Pure and Applied Mathematics, vol. 83. Dekker, New York (1984)

5. Molnár, L., Virosztek, D.: On algebraic endomorphisms of the Einstein gyrogroup. J. Math. Phys. 56, 082302 (2015)

6. Pedersen, G.K.: Some operator monotone functions. Proc. Am. Math. Soc. 36(1), 309-310 (1972)

7. Steele, J.M.: The Cauchy-Schwarz Master Class: An Introduction to the Art of Mathematical Inequalities. MAA Problem Books Series. Cambridge University Press, Cambridge (2008)

8. Ungar, A.A.: Thomas rotation and the parametrization of the Lorentz transformation group. Found. Phys. Lett. 1(1), 57-89 (1988)

9. Ungar, A.A.: Group-like structure underlying the unit ball in real inner product spaces. Results Math. 18(3-4), 355-364 (1990)

10. Ungar, A.A.: Extension of the unit disk gyrogroup into the unit ball of any real inner product space. J. Math. Anal. Appl. 202(3), 1040-1057 (1996)

11. Ungar, A.A.: Analytic Hyperbolic Geometry and Albert Einstein's Special Theory of Relativity. World Scientific, Singapore (2008)

12. Watanabe, K.: A confirmation by hand calculation that the Möbius ball is a gyrovector space. Nihonkai Math. J. 27 99-115 (2016)

13. Watanabe, K.: Orthogonal gyroexpansion in Möbius gyrovector spaces. J. Funct. Spaces 2017, Article ID 1518254 (2017). https://doi.org/10.1155/2017/1518254

14. Watanabe, K.: A Cauchy type inequality for Möbius operations. J. Inequal. Appl. 2018, 97 (2018), https://doi.org/10.1186/s13660-018-1690-2

15. Watanabe, K.: A Cauchy-Bunyakovsky-Schwarz type inequality related to the Möbius addition. J. Math. Inequal. 12(4), 989-996 (2018). https://doi.org/10.7153/jmi-2018-12-75

16. Watanabe, K.: Cauchy-Bunyakovsky-Schwarz type inequalities related to Möbius operations. J. Inequal. Appl. 2019 179 (2019). https://doi.org/10.1186/s13660-019-2132-5

17. Watanabe, K.: Continuous quasi gyrolinear functionals on Möbius gyrovector spaces. J. Funct. Spaces 2020, Article ID 1950727 (2020). https://doi.org/10.1155/2020/1950727

18. Watanabe, K.: On quasi gyrolinear maps between Möbius gyrovector spaces induced from finite matrices. Symmetry 2021(13), 76 (2021). https://doi.org/10.3390/sym13010076

19. Zhu, K.: Spaces of Holomorphic Functions in the Unit Ball. Graduate Text in Mathematics, vol. 226. Springer, New York (2005)

\section{Submit your manuscript to a SpringerOpen ${ }^{\odot}$ journal and benefit from:}

- Convenient online submission

Rigorous peer review

- Open access: articles freely available online

- High visibility within the field

- Retaining the copyright to your article

Submit your next manuscript at $\boldsymbol{~ s p r i n g e r o p e n . c o m ~}$ 